\title{
ON A GAME THEORETIC NOTION OF COMPLEXITY FOR COMPACT CONVEX SETS
}

\author{
EHUD KALAI AND MEIR SMORODINSKY
}

ABSTRACT. The notion of complexity for compact convex sets introduced by Billera and Bixby is considered. It is shown that for $n \geq 3$ there are sets in $R^{n}$ of complexity $n$. Also for $n=3$ the maximal complexity is 3 .

1. Introduction. The following definitions occur in the mathematical model of market games. Let $u_{1}, u_{2}, \cdots, u_{n}$ be $n$ concave continuous real valued functions defined on the $m$-dimensional Euclidean unit cube $I^{m}$.

An allocation (of the commodities) is a matrix $(x)=\left(x_{i}^{j}\right), i=1,2, \cdots$, $m ; j=1,2, \cdots, n$, satisfying the conditions:

(1) $x_{i}^{j} \geq 0$ for every $i$ and $j$, and

(2) $\sum_{j=1}^{n} x_{i}^{j}=1$ for $i=1,2, \cdots, m$.

We define the function $u$ from the set of allocations to $R^{n}$ by

$$
u\left(\left(x_{i}^{j}\right)\right)=\left(u_{1}\left(x_{1}^{1}, x_{2}^{1}, \cdots, x_{m}^{1}\right), \cdots, u_{n}\left(x_{1}^{n}, x_{2}^{n}, \cdots, x_{m}^{n}\right)\right) .
$$

The image of $u$ is a compact subset of $R^{n}$ (see [1]). A subset $V \subset R^{n}$ is attainable if for some $u, V=\left\{p \in R^{n}: p \leq u(x)\right.$ for some allocation $\left.(x)\right\}$. (For $p=\left(p_{1}, \cdots, p_{n}\right)$ and $q=\left(q_{1}, \cdots, q_{n}\right)$, we say that $p \leq q$ if $p_{i} \leq q_{i}$ for $i=1,2, \cdots, n$.$) Another way to define V$ is $V=$ Image $(u)-R_{+}^{n} \cdot\left(R_{+}^{n}=\right.$ $\left.\left\{x=\left(x_{1}, \cdots, x_{n}\right) \mid x_{i} \geq 0, i=1, \cdots, n\right\}.\right)$

Intuitively we think of $n$ traders ("players") in a market of $m$ commodities. $u_{j}\left(x_{1}, x_{2}, \cdots, x_{m}\right)$ represents the utility that the $j$ th player has if he holds the bundle of commodities $\left(x_{1}, x_{2}, \cdots, x_{m}\right)$, where $x_{i}$ represents his portion of the total amount available of the $i$ th commodity.

In a recent paper by Billera and Bixby it was shown that for any compact convex set $C \subset R^{n}$, the set $V=C-R_{+}^{n}$ is attainable for some $\left(u_{1}, u_{2}, \cdots, u_{n}\right)$ on $I^{m}$, where $m$ can be chosen so that $m \leq n(n-1)$. They defined the com-

Received by the editors November 13, 1973 and, in revised form, May 13, 1974 and May 24, 1974.

AMS (MOS) subject classifications (1970). Primary 90A15, 52A20; Secondary 90D12.

Key words and phrases. Compact convex sets, concave utility functions, complexity, market games. 
plexity of $V$ (or $C$ ) to be the minimal $m$ for which this is true, and the complexity of $n(\operatorname{com}(n))$ to be the maximal complexity of an attainable set in $R^{n}$.

It follows from the work of Billera and Bixby that $\operatorname{com}(n) \leq n(n-1)$. It is easy to see that $\operatorname{com}(2)=1$, and in their paper they gave an example of an attainable set in $R^{3}$ with complexity 2 , i.e. $2 \leq \operatorname{com}(3) \leq 6$. One of them conjectured that $\operatorname{com}(n)=n-1$ for all $n$.

In $\$ 2$ we prove that for every $n, n \geq 3$, there is an attainable set of complexity $n$ and, therefore, $\operatorname{com}(n) \geq n$ for $n \geq 3$.

In $\$ 3$ we show that $\operatorname{com}(3)=3$.

We are grateful to Nimrod Megiddo who made valuable comments about the problem.

\section{Complexity in $R^{n}$.}

Lemma 1. Suppose $V_{n}^{*}$ is an attainable set in $R^{n}, n \geq 3$, satisfying the following properties:

(a) the $n+1$ points

$$
\begin{array}{ll}
p_{0}^{*}=(\alpha, \alpha, \cdots, \alpha), & p_{1}^{*}=(1,0, \cdots, 0), \\
p_{2}^{*}=(0,1,0, \cdots, 0), \cdots, & p_{n}^{*}=(0, \cdots, 0,1)
\end{array}
$$

belong to $V_{n}^{*}$ with $0<\alpha<1$;

(b) for each $p=\left(y_{1}, \cdots, y_{n}\right) \in V_{n}^{*}$, if for some $j, 1 \leq j \leq n, y_{j}>a$, then $y_{k}<a$ for every $k \neq j$.

Then $\operatorname{com} V_{n}^{*} \geq n$.

Proof. Assume that $u=\left(u_{1}, \cdots, u_{n}\right)$ are the utility functions on $I^{m}$ that generate $V_{n}^{*}$. Let $(x)=\left(x_{i}^{j}\right)$ be the allocation for which $u(x)=p_{0}^{*}$, i.e. $u_{j}\left(x_{1}^{j}, \cdots, x_{m}^{j}\right)=a$ for $j=1,2, \cdots, n$. Consider the bundle of the $n$th player in this allocation, $\left(x_{1}^{n}, \cdots, x_{m}^{n}\right)$. Let $J$ be the set of indices $i$ for which $x_{i}^{n}=1$. If $J \neq \varnothing$, assume without loss of generality that $J=\{k+1, \cdots, m\}$.

We claim that there is an index $i_{1}, 1 \leq i_{1} \leq k$, such that $x_{i_{1}}^{j}=0$ for every $j, j \neq 1, n$. If not, put $\delta_{i}=\sum_{j \neq 1, n} x_{i}^{j}>0$ for $1 \leq i \leq k$ and $\delta=$ $\min _{1 \leq i \leq n} \delta_{i}$. Consider, for the $n$th player, the set of bundles

$$
B_{n}=\left\{\left(x_{1}^{n}+r_{1}, x_{2}^{n}+r_{2}, \cdots, x_{m}^{n}+r_{m}\right):\left|r_{l}\right|<\delta \text { for } l=1,2, \cdots, m\right\} \cap I^{m}
$$

$B_{n}$ is an open set in the relative topology of $I^{m}$. Since all the bundles of $B_{n}$ can be allocated to the $n$th player without changing the bundle of the 
first player, it follows from property (b) that $u_{n}$ is bounded by $\alpha$ on $B_{n}$. So $u_{n}$ has a local maximum in $B_{n}$ and by concavity it must be a global maximum on $I^{m}$. But this contradicts the requirement that $p_{n}^{*}$ is attained and establishes the truth of the claim.

Observe that $x_{i_{1}}^{\frac{1}{1}}>0$, for if $x_{i_{1}}^{\frac{1}{1}}=0$ then $x_{i_{1}}^{n}=1$ and $i_{1}>k$, which is not the case. Next, repeat the above argument with the 2 nd and $n$th players instead of the 1 st and $n$th players to conclude that there is an index $i_{2}$, $1 \leq i_{2} \leq k$, such that $x_{i_{2}}^{j}=0$ for every $j, j \neq 2, n$, and $x_{i_{2}}^{2}>0$. Notice this implies $i_{1} \neq i_{2}$. Continuing inductively, we find $i_{1}, \cdots, i_{n-1}$ all distinct and all between 1 and $k$ such that $x_{i l}^{j}=0$ for every $j \neq l, n$. This implies immediately that $m \geq k \geq n-1$. Observe that for each player $j \neq n$, there are $n-2$ commodities from which he gets zero quantity.

Suppose that $m=n-1$ so that $J=\varnothing$. If this is the case, assign the roll of player $n$ to player 1 to conclude that there is an index $i_{n}$ such that $x_{i_{n}}^{n}>0$ and $x_{i_{n}}^{j}=0$ for every $j, j \neq 1, n$. This implies that $i_{n}=i_{1}$. But since the choice of player 1 is arbitrary, we can also conclude that $i_{n}=i_{2}$. Since $n \geq 3$, this is a contradiction, and the proof of the Lemma is completed.

Remark. It follows from the proof of Lemma 1 that in the case where $m=n>3$, the allocation for the point $p_{0}^{*}$ is the unit matrix up to a renaming of the commodities.

Theorem 1. The attainable set $\bar{V}_{n}$ in $R^{n}, n \geq 3$, generated by the convex hull of the $n+1$ points

$$
\begin{array}{ll}
p_{0}^{*}=(1 / 2,1 / 2, \cdots, 1 / 2), & p_{1}^{*}=(1,0,0, \cdots, 0), \\
p_{2}^{*}=(0,1,0, \cdots, 0), \cdots, & p_{n}^{*}=(0, \cdots, 0,1)
\end{array}
$$

has complexity $n$.

Proof. To show that the complexity of $\bar{V}_{n}$ is at least $n$, it is enough to show that $\bar{V}_{n}$ satisfies condition (b) of Lemma 1 . Let $p$ be a point in the convex hull of $\left\{p_{0}^{*}, \ldots, p_{n}^{*}\right\}$; then $p=\Sigma_{l=0}^{n} \lambda_{l} p_{l}^{*}$, where $\lambda_{l} \geq 0$ and $\sum_{l=0}^{n} \lambda_{l}=1$. Denoting $p=\left(y_{1}, \cdots, y_{n}\right)$, we then have for $i \neq j$,

$$
y_{i}+y_{j}=\lambda_{0}+\lambda_{i}+\lambda_{j} \leq 1
$$

which proves property (b).

To show that $\operatorname{com} \bar{V}_{n} \leq n$, we exhibit $n$ utility functions from $I^{n}$ to $R$ that generate $\bar{V}_{n}$. Define 


$$
u_{i}\left(x_{1}, \cdots, x_{n}\right)=\frac{1}{2}\left[x_{i}+\min _{1 \leq j \leq n} x_{j}\right] \text { for } i=1, \cdots, n \text {. }
$$

Denote by $\widetilde{V}_{n}$ the attainable set of $u=\left(u_{1}, \cdots, u_{n}\right) . p_{0}^{*}$ is attained at the allocation that assigns to each player $j$ the total amount of the $j$ th commodity. For every player $j, p_{j}^{*}$ is attained at the allocation that assigns to player $j$ the total amount of all the commodities. Therefore $\widetilde{V}_{n} \supset \bar{V}_{n}$. To show the converse inclusion, we start with an arbitrary allocation $x=\left(x_{i}^{j}\right)$ and show that the image of $x$ under $u$ is a point $p=\left(y_{1}, \cdots, y_{n}\right)$ which is less than a point in the convex hull of $\left\{p_{0}^{*}, \cdots, p_{n}^{*}\right\}$. Without loss of generality assume that for every player $j, x_{i}^{j}=\beta_{j}$ if $i \neq j, x_{j}^{j}=\alpha_{j}$ and $\alpha_{j} \geq \beta_{j}$.

Consider the point $p^{\prime}$ which is the image of the allocation that assigns to every player different from 1 and 2 the same amounts as in $x$, assigns to player $1\left(\alpha_{1}-\beta_{1}, 0, \cdots, 0\right)$ and assigns to player $2\left(\beta_{2}+\beta_{1}, \alpha_{2}+\beta_{1}\right.$, $\left.\beta_{2}+\beta_{1}, \cdots, \beta_{2}+\beta_{1}\right)$. Also consider the point $p$ " obtained in the same way as $p^{\prime}$, by interchanging the roles of players 1 and 2 . $p$ is a convex linear combination of $p^{\prime}$ and $p^{\prime \prime}$. Now starting from $p^{\prime}$ and $p^{\prime \prime}$ and making the changes in the bundles of the 2nd player (or 1st in the case of $p^{\prime \prime}$ ) and the 3 rd player, and so on, we finally arrive at allocations where all the off diagonal entries are zero except for one row. We infer that $p$ is a convex linear combination of the images of such allocations of the following form: $x_{j}^{j}=\gamma$ and $x_{i}^{j}=0$ if $i \neq j$ for every $j \neq k ; x_{k}^{k}=1$ and $x_{i}^{k}=1-\gamma$ for $i \neq k$ (where $k$ is the row with the off diagonal nonzero entries). If $\tilde{p}$ is the image of such an allocation, then $\tilde{p}=\left(\tilde{y}_{1}, \cdots, \tilde{y}_{n}\right)$, where $\tilde{y}_{j}=1 / 2 \gamma$ if $j \neq k$ and $\tilde{y}_{k}=1 / 2+(1 / 2-1 / 2 \gamma)=1-1 / 2 \gamma$. So $\tilde{p}=\gamma p_{0}^{*}+(1-\gamma) p_{k}^{*}$, and we conclude that $p$ is a convex linear combination of $\left(p_{0}^{*}, \cdots, p_{n}^{*}\right)$.

3. Complexity in $R^{3}$. In this section we prove that any attainable set $V$ in $R^{3}$ can be attained by three continuous, concave, monotone in each variable, utility functions on $I^{3}$.

Theorem 2. $\operatorname{com}(3)=3$.

Proof. In view of Theorem 1 we have to show only that $\operatorname{com}(3) \leq 3$.

Let $C$ be a compact convex set in $R^{3}$. Assume without loss of generality (see [1, p. 262]) that $\max \left\{y_{1}:\left(y_{1}, y_{2}, y_{3}\right) \in C\right.$ for some $\left.y_{2}, y_{3}\right\}=1$ and that $\min \left\{y_{1}:\left(y_{1}, y_{2}, y_{3}\right) \in C\right.$ for some $\left.y_{2}, y_{3}\right\}=0$. Also assume the same conditions for the 2 nd and 3 rd players. Let $V=C-R_{+}^{3}$. Consider the auxiliary function on $I^{2}$, 


$$
g\left(x_{1}, x_{2}\right)=\max \left\{\delta \in R:\left(x_{1}+\delta, 1-x_{1}+\delta, 1-x_{2}\right) \in V\right\} .
$$

It is easy to verify that $g$ is continuous and concave.

Now we shall define utility functions on $I^{3}$ and prove that these utility functions generate $V$.

$$
\begin{aligned}
& u_{1}\left(x_{1}, x_{2}, x_{3}\right)=x_{1}+g\left(x_{1}, x_{2}\right), \\
& u_{2}\left(x_{1}, x_{2}, x_{3}\right)=x_{1}+g\left(1-x_{1}, x_{3}\right), \\
& u_{3}\left(x_{1}, x_{2}, x_{3}\right)=\min \left\{x_{2}, x_{3}\right\} .
\end{aligned}
$$

Let $\widetilde{V}$ be the attainable set of $u=\left(u_{1}, u_{2}, u_{3}\right)$. To see that $\widetilde{V} \supset V$, consider any point $y=\left(y_{1}, y_{2}, y_{3}\right) \in C$. Choose $\delta_{0}$ such that $y_{1}+\delta_{0}+y_{2}+\delta_{0}$ $=1$. It follows that $0 \leq y_{i}+\delta_{0} \leq 1, i=1$, 2. Consider the allocation

$$
\begin{array}{lll}
x_{1}^{1}=y_{1}+\delta_{0}, & x_{2}^{1}=1-y_{3}, & x_{3}^{1}=0, \\
x_{1}^{2}=y_{2}+\delta_{0}, & x_{2}^{2}=0, & x_{3}^{2}=1-y_{3}, \\
x_{1}^{3}=0, & x_{2}^{3}=y_{3}, & x_{3}^{3}=y_{3} .
\end{array}
$$

A straightforward calculation of $u\left(\left(x_{i}^{j}\right)\right)$ shows that $u\left(\left(x_{i}^{j}\right)\right)=y$.

To prove that $\widetilde{V} \subset V$, let $\left(x_{i}^{j}\right)=x$ be any allocation. It is easy to see that $u_{1}, u_{2}, u_{3}$ are monotonically increasing in each variable, so without loss of generality it can be assumed that $x_{3}^{1}=0, x_{2}^{2}=0, x_{1}^{3}=0$, and $x_{2}^{3}=$ $x_{3}^{3}$. If $\delta=g\left(x_{1}^{1}, 1-x_{3}^{3}\right)$, then there is a point $\left(y_{1}, y_{2}, y_{3}\right) \in V$ such that $y_{1}=x_{1}^{1}+\delta, y_{2}=1-x_{1}^{1}+\delta$ and $y_{3}=x_{3}^{3}$. But these $y_{i}$ 's are the values of the $u_{i}$ 's for the given allocation $x$.

\section{REFERENCE}

1. Louis J. Billera and Robert E. Bixby, A characterization of Pareto surfaces, Proc. Amer. Math. Soc. 41 (1973), 261-267.

DEPARTMENT OF STATISTICS, TEL AVIV UNIVERSITY, TEL AVIV, ISRAEL 